\title{
Thematic Trends in Complementary and Alternative Medicine Applied in Cancer-Related Symptoms
}

\author{
Jose A. Moral-Munoz ${ }^{1,2 \dagger}$, Manuel Arroyo-Morales ${ }^{3}$, Barbara F. Piper ${ }^{4}$, \\ Antonio I. Cuesta-Vargas ${ }^{5}$, Lourdes Díaz-Rodríguez ${ }^{6}$, William C.S. Cho ${ }^{7}$, \\ Enrique Herrera-Viedma ${ }^{8}$, Manuel J. Cobo ${ }^{9}$ \\ ${ }^{1}$ Dept. Nursing and Physiotherapy, University of Cádiz, Cádiz, Spain \\ ${ }^{2}$ Institute of Research and Innovation in Biomedical Sciences of the Province of Cádiz (INiBICA), \\ University of Cádiz, Cádiz, Spain \\ ${ }^{3}$ Dept. Physical Therapy, University of Granada, Granada, Spain \\ ${ }^{4}$ Dept. Nursing, School of Health and Human Services, National University, San Diego, CA, USA \\ ${ }^{5}$ School Clinical Science, Queensland University of Technology, Brisbane, QLD, Australia \\ ${ }^{6}$ Instituto Biosanitario (IBS), University of Granada, Granada, Spain \\ ${ }^{7}$ Hospital Authority, Hong Kong, China \\ ${ }^{8}$ Dept. Computer Science and Artificial Intelligence, University of Granada, Granada, Spain \\ ${ }^{9}$ Dept. of Computer Science and Engineering, University of Cádiz, Cádiz, Spain
}

\section{Abstract}

Purpose: The main goal of this study is to discover the scientific evolution of Cancer-Related Symptoms in Complementary and Alternative Medicine research area, analyzing the articles indexed in the Web of Science database from 1980 to 2013.

Design/Methodology/Approach: A co-word science mapping analysis is performed under a longitudinal framework (1980 to 2013). The documental corpus is divided into two subperiods, 1980-2008 and 2009-2013. Thus, the performance and impact rates, and conceptual evolution of the research field are shown.

Findings: According to the results, the co-word analysis allows us to identify 12 main thematic areas in this emerging research field: anxiety, survivors and palliative care, meditation, treatment, symptoms and cancer types, postmenopause, cancer pain, low back pain, herbal medicine, children, depression and insomnia, inflammation mediators, and lymphedema. The different research lines are identified according to the main thematic areas, centered fundamentally on anxiety and suffering prevention. The scientific community can use this information to identify where the interest is focused and make decisions in different ways.

\footnotetext{
$\dagger$ Corresponding author: Jose A. Moral-Munoz (Email: joseantonio.moral@uca.es).
}

Citation: Jose A. MoralMunoz, Manuel ArroyoMorales, Barbara F. Piper, Antonio I. Cuesta-Vargas, Lourdes Díaz-Rodríguez, William C.S. Cho, Enrique Herrera-Viedma, Manuel J. Cobo (2018). Thematic

Trends in Complementary and Alternative Medicine Applied in Cancer-Related Symptoms.

Vol. 3 No. 2, 2018

pp 1-19

DOI: $10.2478 /$ jdis-2018-0006

Received: Apr. 7, 2018

Revised: May 24, 2018

Accepted: May 28, 2018 


\section{Research Paper}

Research limitation: Several limitations can be addressed: 1) some of the Complementary and Alternative Medicine therapies may not have been included; 2) only the documents indexed in Web of Science are analyzed; and 3) the thematic areas detected could change if another dataset was considered.

Practical implications: The results obtained in the present study could be considered as an evidence-based framework in which future studies could be built.

Originality/value: Currently, there are no studies that show the thematic evolution of this research area.

Keywords Cancer-related symptoms; Complementary and Alternative Medicine; Bibliometrics; Science mapping analysis; Thematic evolution; H-index

\section{Introduction}

A dramatic increase in the number of cancer survivors in older adults is expected to occur over the coming decades (DeSantis et al., 2014). This growing group of survivors will suffer a reduction in their quality of life because of Cancer-Related Symptoms (CRS) and comorbidities and many potentially will die due to the treatment-associated side effects (Cho et al., 2013; Lötzke et al., 2015). Research directed towards clarifying the chronic effects of treatments and identifying risks of late effects during the survivorship phase of cancer is designed to prevent and reduce these late effects and reduce their impact on the continuing downward spiral of disability and decreased quality of life (Alfano et al., 2012). A new integrative evidence-based practice model for providing supportive care in this growing group of cancer survivors is urgently needed.

Complementary and Alternative Medicine (CAM) therapies are being used by approximately one-third of cancer patients (Wyatt, Sikorskii, \& You, 2013). The development of an integrative model (Hu et al., 2015) using an evidence-based approach longitudinally and historically could aid scientists and healthcare providers to not only provide evidence-based care to this emerging group of cancer survivors but could also help guide studies that could be built on the model's existing scientific evidence for the future.

The varying quality and quantity of the conducted research overtime can hamper a detailed analysis of any scientific field. While an expert in the field could develop a detailed analysis, it would nevertheless be a daunting and tedious task to undertake (Perez-Cabezas et al., 2018). For this reason, the use of scientific support tools, such as bibliometrics, can be used to facilitate the analysis of a research field by automatically classifying studies overtime into different themes and topics (Cobo et al., 2011b; Gutiérrez-Salcedo et al., 2017; Van Raan, 2005). Bibliometrics Information Science contributes to the progress of science in many different ways (Martinez et al., 
2014): allowing assessing the progress made, identifying the most reliable sources of scientific publication, laying the academic basis for the evaluation of new developments, identifying major scientific actors, developing bibliometric indexes to assess academic output, etc. Thus, different actors could benefit from this kind of metrics, such as policy-makers, decision-makers, and the general public (Chinchilla-Rodríguez, Miguel, \& Moya-Anegón, 2015; Chinchilla-Rodríguez, Ocaña-Rosa, \& Vargas-Quesada, 2016).

Taking into account the scientific literature, some papers analyzing the bibliometric impact of the CAM research field have been published. Danell and Danell (2009) analyzed the evolution of scientific production in academic journals from 1966 to 2007. Fu et al. (2011) analyzed the document types and the geographical and institutional distribution of the authorship from 1980 to 2009. Tam, Wong, Wong, and Cheung (2012) identified the most frequently cited articles published in the journals indexed in the Integrative and Complementary Medicine Web of Science (WoS) subject category. Moral-Munoz et al. (2014) described the thematic evolution of CAM in the Integrative and Complementary Medicine WoS subject category. Zyoud, Al-Jabi, and Sweileh (2015) analyzed the scientific production of Arab world in CAM. Huang et al. (2015) performed an analysis of the literature of traditional Chinese medicine in PubMed. Furthermore, several papers related to scientific production in cancer have been published. Ugolini et al. (2012) analyzed the scientific production in cancer rehabilitation. Hack et al. (2014) conducted a citation analysis of Canadian psycho-oncology and supportive care. Thonon et al. (2015) analyzed the trends and evolution of French cancer research. Powell et al. (2016) evaluated the 100 most influential manuscripts in gastric cancer. Singh et al. (2016) mapped the breast cancer research in India. Foley et al. (2016) performed an analysis of the 100 most cited articles in esophageal and junctional cancer. Brás et al. (2017) conducted a scientometric study of the oncology research produced by Portugal in the late twentieth century. However, a detailed scientific analysis of CRS and CAM therapies using science mapping has yet to be undertaken.

Therefore, the main aim of the present paper is to analyze the scientific evolution of CRS and CAM research area through the articles retrieved form the WoS database from 1980 to 2013. It is described how a longitudinal Science Mapping Analysis (SMA) (Cobo et al., 2011) was performed to identify the structure, conceptual evolution and future research directions of the analyzed field.

\section{Methodology}

In order to perform the proposed study, a bibliometric analysis is carried out. The method and software SciMAT were used to perform the SMA under a longitudinal framework.

Journal of Data and Information Science

http://www.jdis.org https://www.degruyter.com/view/j/jdis 


\section{Research Paper}

SMA (Cobo et al., 2011) provides a spatial representation of how disciplines, scientific fields, specialties, individual documents and/or authors are related to one other (Small, 1999). It can monitor the evolution of a scientific field and delimit research areas to identify and detail its structure and development over time (Börner, Chen, \& Boyack, 2003; Morris S, Van Der Veer Martens, 2008; Noyons, Moed, \& Luwel, 1999).

On the other hand, co-word analysis is used in SMA to study the conceptual structure of scientific fields, by identifying the most frequent keywords used in the documents (Callon et al., 1983). The open-source software SciMAT (Cobo et al., 2012) was used to carry out the SMA analysis presented in this paper. The followed workflow is shown in Figure 1.

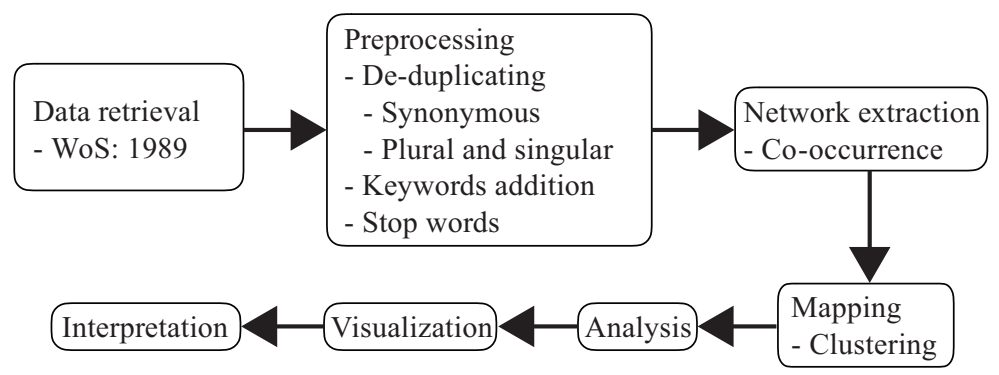

Figure 1. Analysis workflow.

SciMAT is based on a bibliometric approach that establishes four phases with which to analyze the themes and thematic evolution of a research field (Cobo et al., 2015; Moral-Munoz et al., 2018):

1. Research Themes Detection. A normalized bibliometric co-word network of keyword co-occurrence is first built (Callon et al., 1983), using the equivalence index (Callon, Courtial, \& Laville, 1991) as similarity measure. Then, clusters of keywords into topics/themes using a simple centers algorithm (Coulter, Monarch, $\&$ Konda, 1998) are detected. These keyword clustering correspond to centers of interest and/or research problems that are the object of significant interest among researchers.

2. Low Dimensional Space Layout of Research Themes. A spatial layout of research themes is achieved by plotting each research theme using a two-dimensional strategic diagram based on their centrality (degree of interaction of the research theme with other research themes) and density (internal strength of a research theme) rank values (Callon et al., 1991). Once the research themes are mapped into Information Science a two-dimensional space, they can be classified into four groups: motor, basic and 
transversal, emerging or declining, and highly developed and isolated (Cobo et al., 2011).

Themes appearing in the upper-right quadrant are classified as motor themes and are considered to be well developed and important for structuring of a research field. Themes appearing in the lower-right quadrant are classified as basic and transversal and are considered to be important for a research field but are not yet developed. Themes that appear in the lower-left quadrant are classified as emerging or declining and are considered to be weakly or marginally developed. Finally, themes that appear in the upper-left quadrant are categorized as highly developed and isolated and are considered to be well developed but with marginal importance for the field.

3. Discovery of Thematic Areas. The evolution of the previously detected research themes over a set of different time periods is then analyzed to detect the evolution areas in the research field, their origins, and their interrelationships. The evolution of the research themes over the entire study period is determined by the overlapping of clusters between two consecutive periods. To this end, the inclusion index (Sternitzke C. \& Bergmann, 2009) is used to detect the conceptual nexus between research themes from different time periods. It is worth noting that the research themes and their interrelationships might indicate that a particular research theme belongs to a unique thematic area or to more than one thematic area. It could also indicate that a particular research theme cannot be associated with any of the thematic areas identified, and thus could therefore be interpreted as the origin of a new thematic area in the research field.

4. Performance Analysis. The relative contribution of the research themes and thematic areas to the whole research field is measured quantitatively and qualitatively. In this way, the most prominent, productive and highest-impact subfields may be identified. To do this, the following bibliometric indicators are applied to the different detected themes and thematic areas: number of published documents, number of received citations, and h-index (Alonso et al., 2009; Hirsch, 2005).

To carry out the analysis, the data were retrieved from WoS by using three groups of terms related to Cancer, CRS and CAM. The first group of terms referred to the main concepts of cancer and was selected from the Medical Subject Heading $(\mathrm{MeSH})$ thesaurus. These terms included: neoplasm, tumor, neoplasia and cancer. The second group of terms are related to the most common CAM treatments associated with cancer according to Ernst and Casileth (1998) These included terms such as, mind-body, acupuncture, acupressure, etc. The third group of terms included typical symptoms associated with cancer according to Cleeland (2000) (e.g. insomnia, pain, fatigue, etc.). To retrieve the overall number of published documents, the following search strategy was employed:

Journal of Data and Information Science

http://www.jdis.org https://www.degruyter.com/view/j/jdis 


\section{Research Paper}

"TS=(neoplasm\$ OR tumor\$ OR neoplasia OR cancer\$) AND TS=("complementary therap*" OR "complementary medicine" OR "alternative medicine" OR "alternative therap*" OR "integrat* medicine" OR "holistic medicine" OR mind-body OR mindbody OR acupuncture OR acupressure OR music OR massage OR laetrile OR macrobiotic* OR relaxation OR yoga OR "tai chi" OR taichi OR herb* OR beetroot OR homeopath* OR anthroposoph* OR meditation OR hypno* OR reflexology OR iscador) AND TS=("relat* symptom\$” OR insomnia OR pain OR fatigue OR depression \$ OR anxiet \$ OR hypervigilance OR nervousness OR dyspnea OR sleep OR appetite OR cachexia OR shoulder OR nausea OR vomit* OR lymphedema OR "arm morbidity")"

This query retrieved an amount of 1989 documents (articles and reviews) under the period 1980 to 2013. Citation counts up to May 2014 are also used in the analysis.

The raw data was downloaded from WoS as plain text and entered into SciMAT (Cobo et al., 2012). To improve the data quality, a de-duplicating process was applied (the authors keywords and the WoS keywords plus were used as unit of analysis). Words representing the same concept were grouped. Furthermore, some meaningless keywords in this context, such as stop words or words with a very broad and general meaning were removed (e.g. disease, outcomes, system, etc.).

Next, using the SciMAT period manager, the entire study time period (1980 2013) was divided into two, three, and four subperiods, in order to test and establish the adequate number of periods to analyze. In that way, although it is common to use periods during the same time span, in the first years, there were low numbers of researchers and publications. Finally, a first subperiod of twenty-eight years (1980-2008), and a second subperiod of five years were established (2009-2013), since there was not enough document count to obtain an appropriate analysis. It allows us to have enough keywords to perform the analysis previously described.

\section{Results}

In order to introduce the analysis and better understand the subperiod division, the distribution of publications by year between 1980 and 2013 is shown in Figure 2. As we can observe, in the last two years, the number of documents published presents a great growth, i.e. more than 250 documents per year. Therefore, CRS and CAM therapies is a topic which is attracting an increasing interest in the scientific community.

\subsection{Analysis of the Scientific Themes}

Journal of Data and Information Science

To analyze the most notable themes of the research field for each subperiod, a strategic diagram was built using SciMAT. In this diagram, the volume of the 


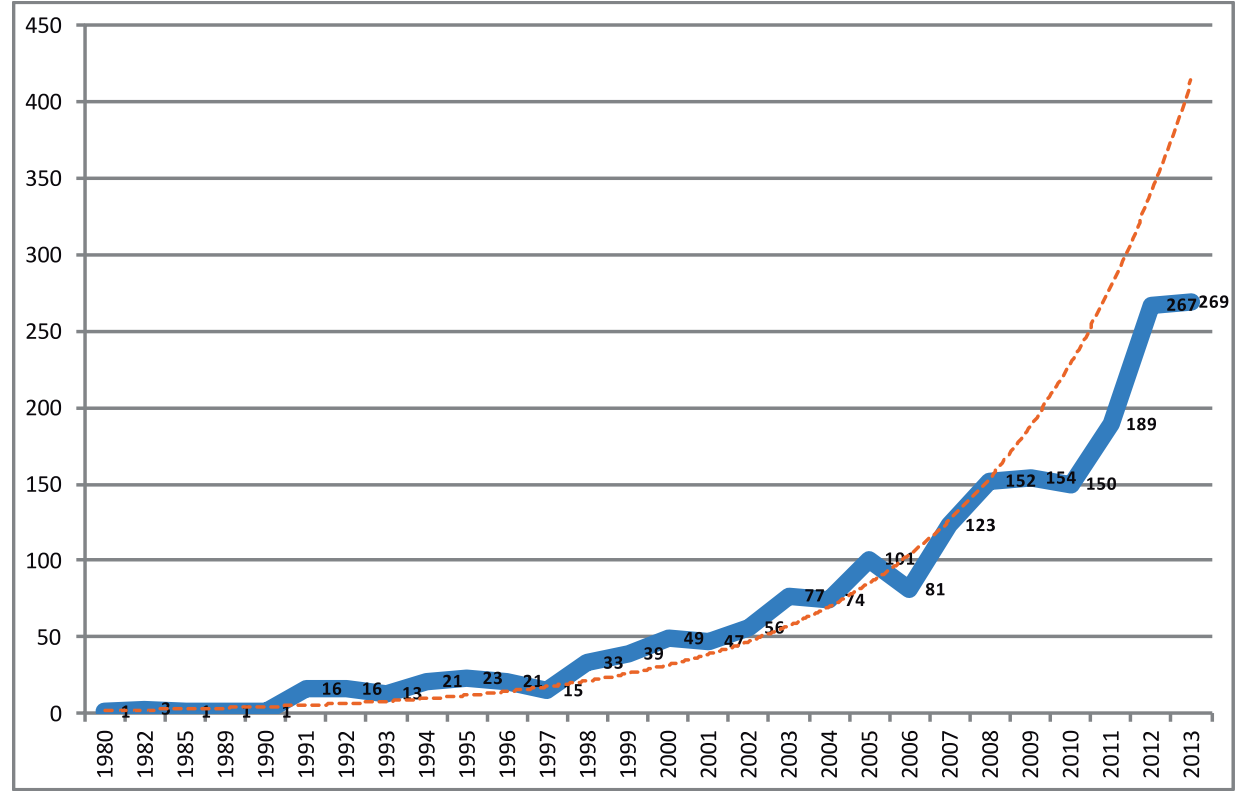

Figure 2. Cancer-related symptoms related to CAM research documents published from 1980 to 2013.

spheres is proportional to the number of documents associated with each theme. In brackets, the number of citations associated with each theme is also depicted. The strategic diagram and performance analysis are analyzed together.

\subsubsection{First Period of Analysis (1980-2008)}

According to the strategic diagram shown in Figure 3, some observations can should be remarked:

The motor themes Anxiety, Meditation, Postmenopausal Women and Acupressure represent the best performance rates with the highest h-index and citations count. The theme Anxiety is related to two main terms, pain and depression. This theme is composed of causes of anxiety and its related treatment (e.g. relaxation, hypnosis, aromatherapy, massage). The theme Meditation is related to the reduction of anxiety and stress. An important theme in relation to meditation is Quality of Life. Postmenopausal Women, is related to characteristic symptoms of the postmenopause (e.g. hot-flashes, vasomotor symptoms) and its therapies (e.g. hormone replacement therapy, black-cohosh). Lastly, the theme Acupressure is closely related to acupuncture and its thematic network is composed of words associated with CRS such as nausea, vomiting and morning sickness. This theme also illustrates the research interest in using acupuncture to treat these symptoms.

Journal of Data and Information Science http://www.jdis.org https://www.degruyter.com/view/j/jdis 


\section{Research Paper}

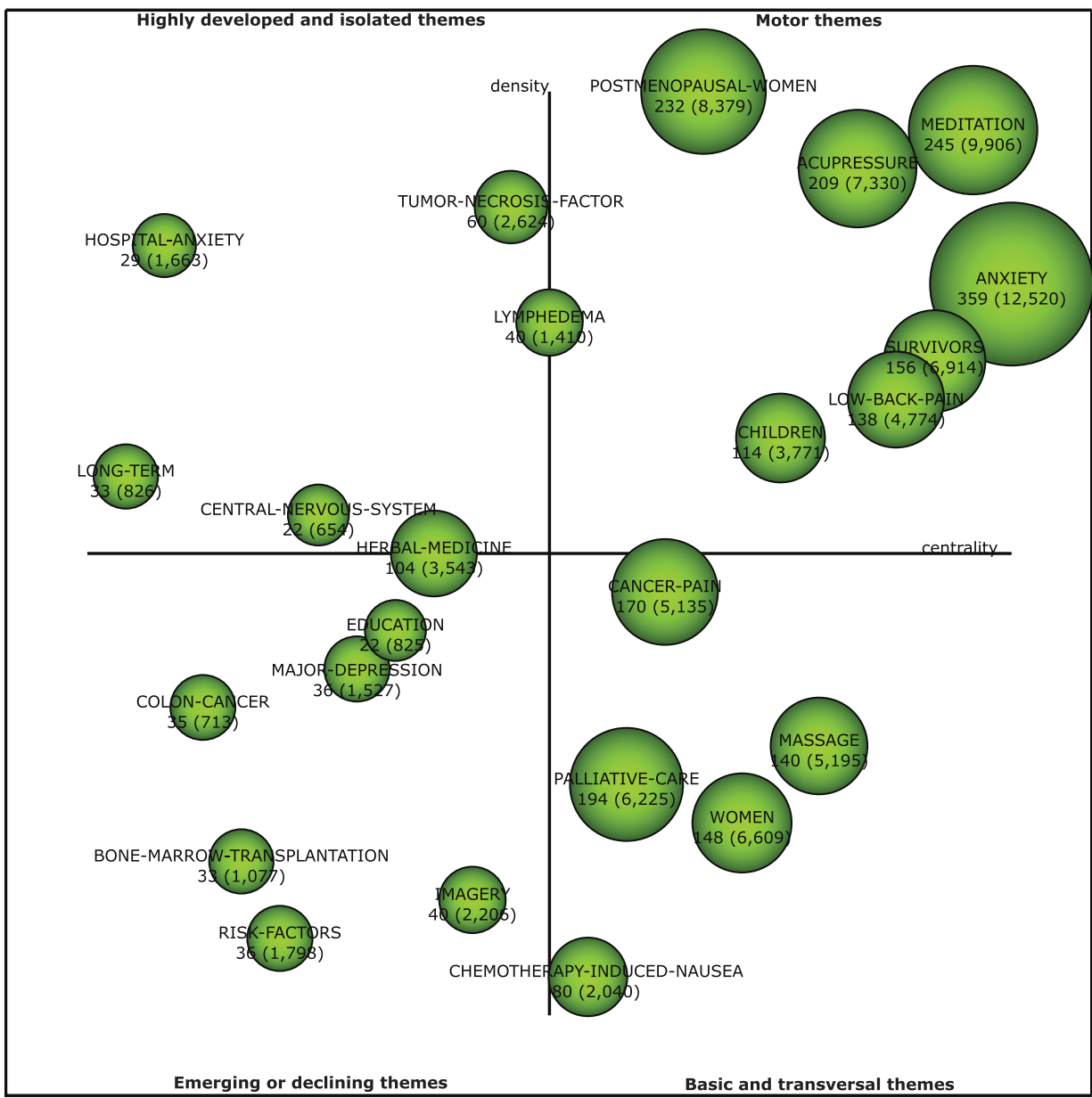

Figure 3. Strategic diagram for the subperiod 1980-2008.

The motor themes Survivors and Low Back Pain, although with a fewer number of documents, present an adequate citation rates and an h-index. The theme Survivors is composed of terms related to psychological aspects and themes such as Radiotherapy or Morbidity in patients who suffer cancer and who are still alive. The theme Low Back Pain mainly addresses pain management of this body part with special attention given to acupuncture and physiotherapy.

The basic and transversal themes Palliative Care, Cancer Pain, Women and Massage present similar good performance rates. The theme Women has a higher

Journal of Data and number of citations and h-index but with fewer documents when compared to Information Science Palliative Care. The theme Palliative Care is focused on relieving and preventing 
patient suffering. This theme is related to music-therapy, morphine and electricalnerve-stimulation treatments; all treatments designed to reduce pain and suffering. The theme Cancer Pain addresses painful feelings experienced by cancer patients and the medications that are designed to reduce these feelings. The theme Women is a general theme about cancers that primarily affect females and it encompasses mastectomy and exercise. Finally, the theme Massage is related to the use of this technique to alleviate selected CRS and examining its effects on immune function.

The basic and transversal theme Chemotherapy-Induced Nausea underscores the importance of this symptom. It is composed of the theme Electro-Acupuncture that suggests that this form of treatment is widely studied as a therapy for nausea.

The isolated or emerging/declining theme Herbal Medicine primarily addresses use of plants as treatment. Two herbal extracts were identified: hypericum-perforatum and mistletoe; the former is used as a depressive treatment, the latter is used in cancer treatment. Furthermore, the presence of the term rats suggests that this kind of therapy is important in experimental research conducted in animal studies.

The emerging/declining theme Imagery has a small quantity of documents but was more frequently cited than were other themes with more citations like Chemotherapy-Induced Nausea, which had twenty more citations. Imagery is a traditional mind-body technique that is considered to be a form of hypnosis and is related to behavioral treatment.

The isolated and highly developed theme Lymphedema illustrates the importance of this symptom in this research field. It is also remarkable the inclusion of evidencebased medicine.

\subsubsection{Second Period of Analysis (2009-2013)}

Several observations can be made about the strategic diagrams (Figure 4).

The motor themes Anxiety and Mindfulness have the highest impact rates. The theme Mindfulness is related to meditation and mind-body medicine. It appears in relationship to stress and cognitive therapy. It has a remarkable association to Quality of Life with a high volume of citations. In this capacity, Anxiety is related to stress and stress reducing forms of treatments such as massage or music-therapy. On the other hand, Hot Flashes is important in this research field and is related to similar themes such as postmenopausal women noted in the in the previous subperiod. The term Breast Cancer has a high volume of citations related to this theme.

The motor theme Fatigue is a new theme documented during this period and is related to two main concepts. One concept includes a group of citations that relate fatigue to being a physical activity consequence. The other group of citations relate fatigue to depression and sleep disturbance in cancer survivors.

Journal of Data and Information Science

http://www.jdis.org https://www.degruyter.com/view/j/jdis 


\section{Research Paper}

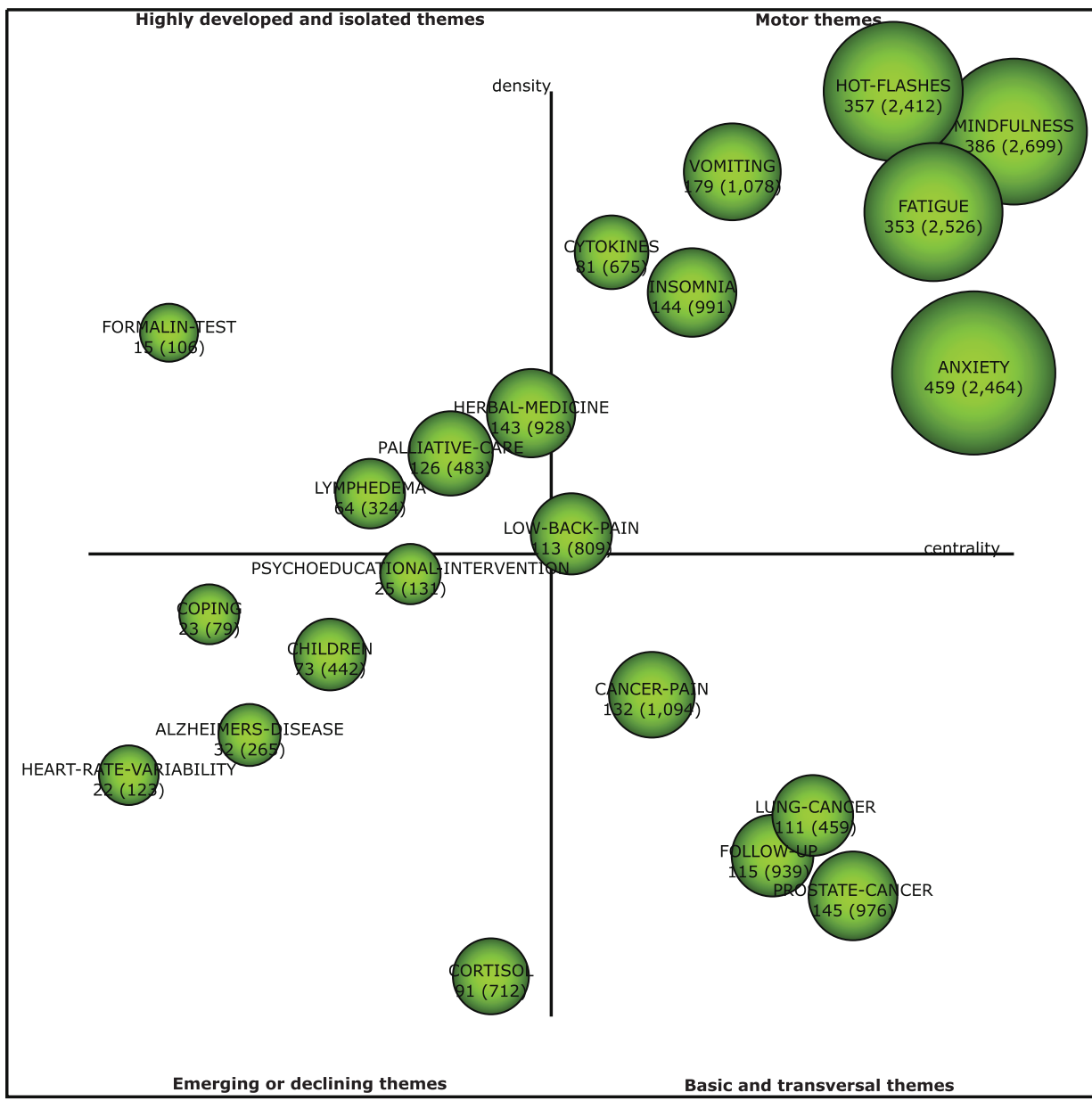

Figure 4. Strategic diagram for the subperiod 2009-2013.

The motor themes of Vomiting and Insomnia are related to these important symptoms. The theme Vomiting is closely related to nausea, specifically as it is associated with being chemotherapy- induced. It is highlighted by documents that address prevention and treatment of same (e.g. acupressure). The theme Insomnia is another symptom associated with a sleep disorder; it is related to depression and it is identified or associated with different alternative therapies (hypnotherapy, cognitive behavioral therapy).

The motor theme Low Back Pain appears in this period related to multiple

Journal of Data and sclerosis, osteoarthritis and fibromyalgia. Furthermore, it is interesting that it is also Information Science related to evidence-based medicine. Finally, the theme Cytokines, despite a low 
number of articles shows a remarkably high citation rate and h-index. It is mainly related to meditators involved in anti-inflammatory processes.

The basic or transversal theme Prostate Cancer is related to other kind of cancers (e.g. colorectal cancer, neck cancer) and is composed of documents that mainly focus on dietary supplements, radiotherapy, psychological distress and hospital anxiety.

The basic or transversal theme Cancer Pain appeared in the previous subperiod, but in this recent period it is related to newer concepts such as social-support and perceptions, which suggest new research interests; furthermore, the term ElectroAcupuncture seems to be an alternative therapy studied in cancer pain.

The emerging/declining theme Cortisol is an emerging theme in with adequate performance rates. It is related also to immune system and inflammation processes.

The isolated theme Herbal Medicine appears related to two specific cancers, gastric cancer and lung cancer. Also, the theme Palliative Care is present too in this period. It should be noticed its association with terms like family and familycaregivers, and documents focused in home-based care.

\subsection{Thematic Evolution of the Model Over Time}

The thematic evolution over time in the field of CRS and CAM can now be examined. Twelve thematic areas have been identified over these two subperiods of time (Table 1).

Table 1. Performance measures for the detected areas.

\begin{tabular}{lcccc}
\hline \multicolumn{1}{c}{ Theme Name } & $\begin{array}{c}\text { Number of } \\
\text { Documents }\end{array}$ & $\begin{array}{c}\text { Number of } \\
\text { Citations }\end{array}$ & h-Index & g-index \\
\hline ANXIETY & 969 & 19,156 & 66 & 104 \\
SURVIVORS-AND-PALLIATIVE-CARE & 832 & 17,549 & 64 & 100 \\
MEDITATION & 669 & 12,837 & 54 & 89 \\
TREATMENT-SYMPTOMS-AND-CANCER-TYPES & 638 & 12,182 & 52 & 86 \\
POSTMENOPAUSE & 589 & 10,791 & 51 & 80 \\
CANCER-PAIN & 302 & 6,229 & 38 & 67 \\
LOW-BACK-PAIN & 272 & 6,232 & 41 & 69 \\
HERBAL-MEDICINE & 247 & 4,471 & 36 & 58 \\
CHILDREN & 187 & 4,213 & 35 & 58 \\
DEPRESSION-AND-INSOMNIA & 180 & 2,518 & 26 & 45 \\
INFLAMMATION-MEDIATORS & 141 & 3,299 & 30 & 54 \\
LYMPHEDEMA & 135 & 2,498 & 27 & 46 \\
\hline
\end{tabular}

In the thematic evolution diagram shown in Figure 5, each column corresponds to a period, 1980-2008 and 2009-2013 respectively. The volume of the spheres is proportional to the number of documents associated with each theme. Solid lines mean that the linked themes share the name: both themes have the same name, or

Journal of Data and Information Science

http://www.jdis.org https://www.degruyter:com/view/j/jdis 


\section{Research Paper}

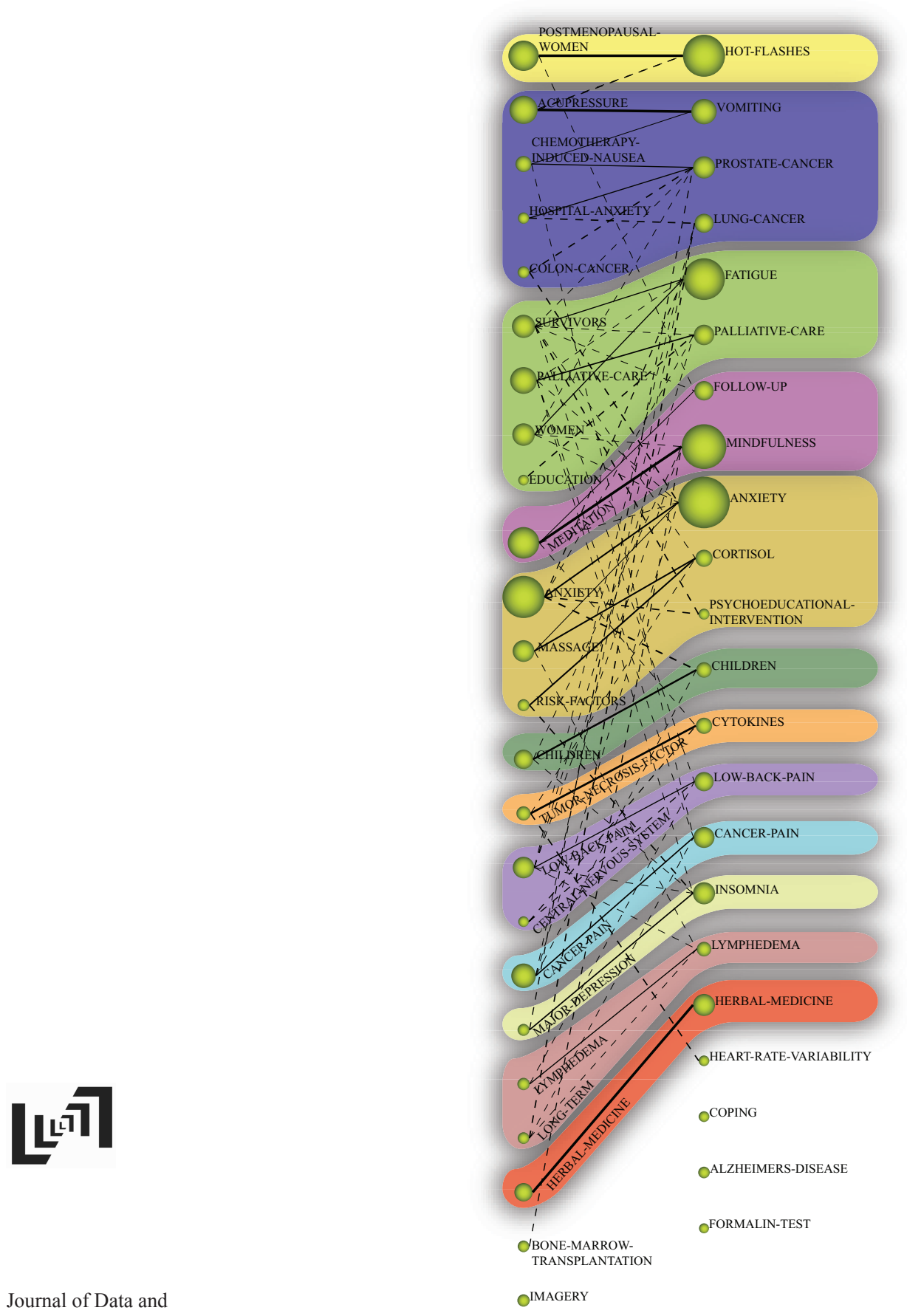

Information Science

Figure 5. Thematic evolution of Cancer-related Symptoms in Complementary and Alternative Medicine (1980-2013). 
the name of one of the themes is part of the other theme. A dotted line means that the themes share elements that are not the name of the theme. The color-shadows group the themes that belong to the same thematic area. Some themes do not have a shadow, because these themes do not belong to any thematic area. In Table 1, their respective global quantitative and impact measures are provided.

To analyze the evolution of this field, Figure 5 and Table 1 show several findings that need to be highlighted to point out thematic relationships to composition, structure and impact.

Thematic Composition. The thematic area Anxiety is composed of motor and emerging/declining themes. It includes in both periods one important motor theme, called a thematic area. Also, this thematic area incorporates two new emerging themes in the second period. Survivors and Palliative Care starts with a motor and two basic themes and it develops into a motor and an isolated theme.

Three thematic areas are mainly composed by motor themes: Meditation, Postmenopause and Low Back Pain. These are consolidated into important thematic areas within the research field.

Treatment, Symptoms, and Cancer Types is the thematic area that presents the greatest number of themes. In the first subperiod it starts from a motor, a basic, an isolated and an emerging theme and it converges into a motor and two basic themes. The thematic area Cancer Pain is consolidated as a basic theme throughout the two time periods.

Two thematic areas are mainly composed by isolated themes: Herbal Medicine and Lymphedema. They are consolidated as isolated themes throughout the two time periods.

The thematic area Children starts from a motor theme and in the second subperiod it converges to a declining theme.

Two thematic areas converge to a motor theme: Depression and Insomnia and Inflammation Mediators. The former includes in its origin just an emerging theme, and it converges to a motor theme. The latter starts as an isolated theme and it develops into a motor theme.

Structural Evolution. The research field presents a great cohesion, due to the fact that the majority of detected themes are grouped into a thematic area. Furthermore, most of these evolutions are part of a thematic nexus. Some very recent themes (e.g. Coping, Alzheimer's Disease, and Formalin Test) could not be identified with any thematic area. These could be considered to be emergent new thematic areas. In general, the thematic areas present a growing pattern of research over time. The number of documents in the second period is greater than in the period before.

Performance and Impact Indicators. Regarding the performance and impact indicators some aspects should be highlighted. The thematic area Anxiety is the

Journal of Data and Information Science

http://www.jdis.org https://www.degruyter:com/view/j/jdis 


\section{Research Paper}

most important theme in terms of the number of documents and citations, with the highest h-index and the best impact scores. On the other hand, Survivor and Palliative Care is the second thematic area with higher performance rates. The thematic areas Meditation, Treatment, Symptoms, Cancer Types, and Postmenopause also present a high citation rates and h-index. The thematic areas Menopause and Anxiety are significant, as they achieve a high growth in the second period.

\section{Discussion}

In this paper, a bibliometric analysis of CRS and CAM has been developed using SMA. As stated above, bibliometrics allows to analyze a research are in several ways (Santisteban-Espejo et al., 2018). Thus, in relation to the results obtained, some findings can be highlighted. The first remarkable finding is that the research field has attracted the interest of the scientific community throughout years. It is observed in the great growth of publications and citations received.

According to the analysis, it identifies for the first period twelve different main themes in CRS and CAM. All these findings are supported by the current scientific literature, which highlight the importance of these research lines. Anxiety is an important symptom as are its treatments. In the second period research is more focused on the physiological changes involved in anxiety. It is in relation to recent findings, where the repercussion of the anxiety in the emotional competences is highlighted (Balasooriya-Smeekens, Walter, \& Scott, 2015; Baudry et al., 2018). Survivor and Palliative Care emphasizes healthcare that cancer patients receive following treatment and is highly focused on fatigue suffered by patients (Kassianos et al., 2018). Meditation is focused on the benefit of using this form of therapy in stressed patients and it is important to the concept of quality of life related to this thematic area (Haller et al., 2017). Treatment, Symptoms, and Cancer Types is a thematic area covering different kinds of topics: treatments (e.g. acupressure), symptoms (e.g. vomiting) and cancer types (e.g. prostate cancer). Postmenopause is mainly related to symptoms and hormonal changes that occur during postmenopause. It is also related to the term breast cancer in both periods (Rosner et al., 2015). Cancer Pain is mainly composed of themes related to treatments; in the first period it is centered on drugs and opioids, and in the second period it is focused on alternative therapies (Chiu, Hsieh, \& Tsai, 2017; Kane et al., 2017). Low Back Pain is mainly focused on physiotherapy and therapies to reduce the pain in both periods (Ross \& Bayer, 2005). Herbal Medicine is related to the use of herbal extracts to treat some CRS, and the presence of documents where herbal medicine is tested in animals (Lau et al., 2016). Children in the first period is related to different alternative therapies (e.g. hypnosis, imagery) and some symptoms (e.g. stress, behavior change) (Italia et al., 2015). In the second period it is less important 
and is related to more general terms. Depression and Insomnia is focused on those two CRS and their alternative treatments such as hypnotherapy and cognitive behavioral therapy (Cha et al., 2017). Inflammation Mediators is related to cytokines involved in immune processes, and is mainly focused on tumor necrosis factor (Fernandes et al., 2015). Lymphedema is primarily related to breast cancer and the physical treatment of this CRS (Shaitelman et al., 2015).

Regarding the thematic evolution, the thematic area Anxiety obtains the best impact index with the highest values in terms of the number of citations and h-index. This means that Anxiety is a growing research area with a good rate of published documents that captures the attention of the CRS and CAM research field. The thematic area Survivors and Palliative Care is another highly developed thematic area with a great number of documents published. The amount of documents published and the different issues related to it suggest that preventing the suffering of patients with alternative therapies is a major focus in this field.

Nevertheless, some study limitations can be highlighted. First, the most used CAM therapies, according to the literature, are analyzed, although some of them may not have been included. Second, the use of WoS as bibliographic source implies that articles not indexed in the database have not been analyzed. If they would be taken into account, the detected thematic areas could change.

\section{Conclusion}

Based on these study results, the following conclusions can be made. The scientific community can now identify thematic areas on which attention has been focused over time and others that are growing in terms of current research interest. The citations and scientific production in CRS and CAM are primarily focused on two large topics, anxiety and the prevention of suffering in patients following cancer treatment. There are other thematic areas that are important in terms of research interest. These include meditation, symptoms as vomiting or nausea and postmenopause. These results suggest that this research field is growing rapidly and will continue attracting more research in the future. The study findings can be used to provide an historical perspective on research conducted in this emerging field and a scientific evidence-based practice model that could be used to develop future research.

\section{Acknowledgements}

This work has been supported by the Andalusian Excellence Project TIC-5991, and Spanish National Project TIN2016-75850-R. J.A. Moral-Munoz held an FPU scholarship (AP2012-1789) from the Spanish Ministry of Education.

Journal of Data and Information Science

http://www.jdis.org https://www.degruyter.com/view/j/jdis 


\section{Research Paper}

\section{Author Contributions}

Jose A. Moral-Munoz (joseantonio.moral@uca.es) conceived and designed the analysis, contributed data or analysis tools, performed the analysis and wrote the paper. Manuel J. Cobo (manueljesus.cobo@uca.es) and Enrique Herrera-Viedma (viedma@decsai.ugr.es) conceived and designed the analysis, contributed data or analysis tools. Manuel Arroyo-Morales (marroyo@ugr.es), Barbara F. Piper (bpiper@nu.edu), Antonio I. Cuesta-Vargas (acuesta@uma.es), Lourdes DíazRodríguez (cldiaz@ugr.es), and William C.S. Cho (williamcscho@gmail.com) conceived and designed the analysis.

\section{References}

Alfano, C.M., Ganz, P.A., Rowland, J.H., \& Hahn, E.E. (2012). Cancer survivorship and cancer rehabilitation: revitalizing the link. Journal of Clinical Oncology, 30(9), 904-906.

Alonso, S., Cabrerizo, F.J., Herrera-Viedma, E., \& Herrera, F. (2009). H-index: A review focused in its variants, computation and standardization for different scientific fields. Journal of Infometrics, 3(4), 273-289.

Balasooriya-Smeekens, C., Walter, F.M., \& Scott, S. (2015). The role of emotions in time to presentation for symptoms suggestive of cancer: A systematic literature review of quantitative studies. Psycho-Oncology, 24(12), 1594-1604.

Baudry, A.S., Lelorain, S., Mahieuxe, M., \& Christophe, V. (2018). Impact of emotional competence on supportive care needs, anxiety and depression symptoms of cancer patients: a multiple mediation model. Supportive Care in Cancer, 26(1), 223-230.

Börner, K., Chen, C., \& Boyack, K.W. (2003). Visualizing knowledge domains. Annual Review of Information Science and Technology, 37(1), 179-255.

Brás, O.R., Cointet, J.-P., Cambrosio, A., David, L., Nunes, J.A., Cardoso, F., \& Jerónimo, C. (2017). Oncology research in late twentieth century and turn of the century Portugal: a scientometric approach to its institutional and semantic dimensions. Scientometrics, 113(2), $867-888$.

Callon, M., Courtial, J.P., \& Laville, F. (1991). Co-word analysis as a tool for describing the network of interactions between basic and technological research: the case of polymer chemistry. Scientometrics, 22(1), 155-205.

Callon, M., Courtial, J.P., Turner, W.A., \& Bauin, S. (1983). From translation to problematic networks: An introduction to co-word analysis. Social Science Information, 22(2), 191-235.

Cha, K.M., Chung, Y.K., Lim, K.Y., Noh, J.S., Chun, M., Hyun, S.Y., Kang, D.R., Oh, M.J., \& Kim, N.H. (2017). Depression and insomnia as mediators of the relationship between distress and quality of life in cancer patients. Journal of Affective Disorders, 217, 260-265.

Chinchilla-Rodríguez, Z., Ocaña-Rosa, K., \& Vargas-Quesada, B. (2016). How to combine research guarantor and collaboration patterns to measure scientific performance of countries in scientific fields: Nanoscience and Nanotechnology as a Case Study. Frontiers in Research Metrics and Analytics, 1, 2.

Journal of Data and Information Science

Chinchilla-Rodríguez, Z., Miguel, S., \& Moya-Anegón, F. De. (2015). What factors affect the visibility of argentinean publications in humanities and social sciences in scopus? Some evidence beyond the geographic realm of research. Scientometrics, 102(1), 789-810. 
Thematic Trends in Complementary and Alternative Medicine Applied in

Jose A. Moral-Munoz et al. Cancer-Related Symptoms

Chiu, H.Y., Hsieh, Y.J., \& Tsai, P.S. (2017). Systematic review and meta-analysis of acupuncture to reduce cancer-related pain. European Journal of Cancer Care, 26(2), 1-17.

Cho, H., Mariotto, A.B., Mann, B.S., Klabunde, C.N., \& Feuer, E.J. (2013). Assessing noncancer-related health status of US cancer patients: other-cause survival and comorbidity prevalence. American Journal of Epidemiology, 17(3), 339-349.

Cleeland, C.S. (2000). Cancer-Related Symptoms. Seminars in Radiation Oncology, 10(3), 175190.

Cobo, M.J., López-Herrera, A.G.G., Herrera-Viedma, E., \& Herrera, F. (2012). SciMAT: A new science mapping analysis software tool. Journal of the American Society for Information Science and Technology, 63(8), 1609-1630.

Cobo, M.J., López-Herrera, A.G., Herrera-Viedma, E., \& Herrera, F. (2011a). An approach for detecting, quantifying, and visualizing the evolution of a research field: A practical application to the fuzzy sets theory field. Journal of Infometrics, 5(1), 146-166.

Cobo, M.J., López-Herrera, A.G., Herrera-Viedma, E., \& Herrera, F. (2011b). Science mapping software tools: Review, analysis, and cooperative study among tools. Journal of the Association for Information Science and Technology, 62(7), 1382-1402.

Cobo, M.J., Martinez, M.A., Gutierrez-Salcedo, M., Fujita, H., \& Herrera-Viedma, E. (2015). 25 years at knowledge-based systems: A bibliometric analysis. Knowledge-Based Systems, 80, $3-13$.

Coulter, N., Monarch, I., \& Konda, S. (1998). Software engineering as seen through its research literature: A study in co-word analysis. Journal of the American Society for Information Science, 49(13), 1206-1223.

Danell J.A.B., \& Danell, R. (2009). Publication activity in complementary and alternative medicine. Scientometrics, 80(2), 539-551.

DeSantis, C.E., Lin, C.C., Mariotto, A.B., Siegel, R.L., Stein, K.D., Kramer, J.L., Alteri, R, Robbins, A.S., \& Jemal, A. (2014). Cancer treatment and survivorship statistics. Cancer Journal for Clinicians, 64(4), 252-271.

Ernst, E., \& Casileth, B.R. (1998). The Prevalence of Complementary/Alternative Medicine in Cancer. American Cancer Society, 83(4), 777-782.

Fernandes, J.V., Cobucci, R.N.O., Jatobá, C.A.N., de Medeiros Fernandes, T.A.A., de Azevedo, J.W.V., \& de Araújo, J.M.G. (2015). The role of the mediators of inflammation in cancer development. Pathology and Oncology Research, 21(3), 527-534.

Foley, K.G., Powell, A., Lewis, W.G., \& Roberts, S.A. (2016). The 100 most cited articles investigating the radiological staging of oesophageal and junctional cancer: a bibliometric analysis. Insights into Imaging, 7(4), 619-628.

Fu, J.Y., Zhang, X., Zhao, Y.H., Huang, M.H., \& Chen, D.Z. (2011). Bibliometric analysis of complementary and alternative medicine research over three decades. Scientometrics, 88(2), 617-626.

Gutiérrez-Salcedo, M., Martínez, M.Á., Moral-Munoz, J.A., Herrera-Viedma, E., \& Cobo, M.J. (2017). Some bibliometric procedures for analyzing and evaluating research fields. Applied Intelligence, 48(5), 1275-1287.

Hack, T.F., Crooks, D., Plohman, J., \& Kepron, E. (2014). Citation analysis of Canadian psychooncology and supportive care researchers. Supportive Care in Canceri, 22, 315-324.

Journal of Data and Information Science

http://www.jdis.org https://www.degruyter:com/view/j/jdis 


\section{Research Paper}

Haller, H., Winkler, M.M., Klose, P., Dobos, G., Kümmel, S., \& Cramer, H. (2017). Mindfulnessbased interventions for women with breast cancer: An updated systematic review and meta-analysis. Acta Oncologica, 56(12), 1665-1676.

Hirsch, J. (2005). An index to quantify an individuals scientific research output. Proceeding of the National Academy of Sciences, 102, 15572-16569.

Hu, X.Y., Lorenc, A., Kemper, K., Liu, J.P., Adams, J., \& Robinson, N. (2015). Defining integrative medicine in narrative and systematic reviews: A suggested checklist for reporting. European Journal of Integrative Medicine, 7(1), 76-84.

Huang, Y., Zhou, M., Deng, Q., Zhang, J., Zhou, P., \& Shang, X. (2015). Bibliometric analysis for the literature of traditional Chinese medicine in PubMed. Scientometrics, 105(1), 557-566.

Italia, S., Brand, H., Heinrich, J., Berdel, D., von Berg, A., \& Wolfenstetter, S.B. (2015). Utilization of complementary and alternative medicine (CAM) among children from a German birth cohort (GINIplus): patterns, costs, and trends of use. BMC Complementary and Alternative Medicine, 15(1), 49.

Kane, C.M., Mulvey, M.R., Wright, S., Craigs, C., Wright, J.M., \& Bennett, M.I. (2017). Opioids combined with antidepressants or antiepileptic drugs for cancer pain: Systematic review and meta-analysis. Palliative Medicine, 26921631771182.

Kassianos, A.P., Ioannou, M., Koutsantoni, M., \& Charalambous, H. (2018). The impact of specialized palliative care on cancer patients' health-related quality of life: a systematic review and meta-analysis. Supportive Care in Cancer, 26(1), 61-79.

Lau, C.H.Y., Wu, X., Chung, V.C.H., Liu, X., Hui, E.P., Cramer, H., Lauche, R., Lau, A.Y., Sit, R.S., Ng, B.F., \& Wu, J.C.Y. (2016). Acupuncture and related therapies for symptom management in palliative cancer care: Systematic review and meta-analysis. Medicine, 95(9), e2901.

Lötzke, D., Recchia, D.R., Kröz, M., Reif, M., Gutenbrunner, C., Zerm, R., Nikolaou, A., Berger, B., \& Büssing, A. (2015). The influence of a multimodal therapy concept on health-related quality of life in breast cancer survivors with cancer-related fatigue in comparison to a standard aerobic therapy. European Journal of Integrative Medicine, 7, 14.

Martinez, M.A., Herrera, M., Lopez-Gijon, J., \& Herrera-Viedma, E. (2014). H-classics: Characterizing the concept of citation classics through h-index. Scientometrics, 98(3), 1971-1983.

Moral-Munoz, J.A., Arroyo-Morales, M., Herrera-Viedma, E., \& Cobo, M.J. (2018). An overview of thematic evolution of physical therapy research area from 1951 to 2013. Frontiers in Research Metrics and Analytics, 3, 13.

Moral-Munoz, J.A., Cobo, M.J., Peis, E., Arroyo-Morales, M., \& Herrera-Viedma, E. (2014). Analyzing the research in Integrative \& Complementary Medicine by means of science mapping. Complementary Therapies in Medicine, 22(2), 409-418.

Morris, S., \& Van Der Veer Martens, B. (2008). Mapping research specialities. Annual Review of Information Science and Technology, 42 (1), 213-295.

Noyons, E.C.M., Moed, H.F., \& Luwel, M. (1999). Combining mapping and citation analysis for evaluative bibliometric purposes: A bibliometric study. Journal of the American Society for Information Science, 50(2), 115-131.

Journal of Data and Information Science

Perez-Cabezas, V., Ruiz-Molinero, C., Carmona-Barrientos, I., Herrera-Viedma, E., Cobo, M.J., \& Moral-Munoz, J.A. (2018). Highly cited papers in Rheumatology: Identification and conceptual analysis. Scientometrics, in press. 
Powell, A.G., Hughes, D.L., Wheat, J.R., \& Lewis, W.G. (2016). The 100 most influential manuscripts in gastric cancer: A bibliometric analysis. International Journal of Surgery (London, England), 28, 83-90.

Rosner, B., Eliassen, A.H., Toriola, A.T., Hankinson, S.E., Willett, W.C., Natarajan, L., \& Colditz, G.A. (2015). Short-term weight gain and breast cancer risk by hormone receptor classification among pre- and postmenopausal women. Breast Cancer Research and Treatment, 150(3), 643-653.

Ross, M.D., \& Bayer, E. (2005). Cancer as a cause of low back pain in a patietn seen in a direct access physical therapy setting. J Orthop Sports Phys Ther, 35(10), 651-658.

Santisteban-Espejo, A., Campos, F., Martin-Piedra, L., Durand-Herrera, D., Moral-Munoz, J.A., Campos, A., \& Martin-Piedra, M.A. (2018). Global tissue engineering trends: A scientometric and evolutive study. Tissue Engineering Part A, in press.

Shaitelman, S.F., Cromwell, K.D., Rasmussen, J.C., Stout, N.L., Armer, J.M., Lasinski, B.B., \& Cormier, J.N. (2015). Recent progress in the treatment and prevention of cancer-related lymphedema. CA: A Cancer Journal for Clinicians, 65(1), 55-81.

Singh, N., Handa, T.S., Kumar, D., \& Singh, G. (2016). Mapping of breast cancer research in India: a bibliometric analysis. Current Science (00113891), 110(7), 1178-1183.

Small, H. (1999). Visualizing science by citation mapping. Journal of the American Society for Information Science, 50, 799-813.

Sternitzke C.; Bergmann, I. (2009). Similarity measures for document mapping: A comparative study on the level of an individual scientist. Scientometrics, 3(4), 113-130.

Tam, W.W.S., Wong, E.L.Y., Wong, F.C.Y., \& Cheung, A.W.L. (2012). Citation classics in the integrative and complementary medicine literature: 50 frequently cited articles. European Journal of Integrative Medicine, 4(1), e77-e83.

Thonon, F., Saghatchian, M., Nerfie, A., \& Delaloge, S. (2015). Tendances et évolutions de la recherche française sur le cancer du sein: étude bibliométrique. Bulletin Du Cancer, 102(5), 417-427.

Ugolini, D., Neri, M., Cesario, A., Bonassi, S., Milazzo, D., Bennati, L., Lapenna, L.M., \& Pasqueletti, P. (2012). Scientific production in cancer rehabilitacion grows higher: A bibliometric analysis. Supportive Care in Cancer, 20, 1629-1638.

Van Raan, A.F.J. (2005). Handbook of quantitative science and technology research, chapter Measuring Science. Springer Netherlands, 19-50.

Wyatt, G., Sikorskii, A., \& You, M. (2013). Self-reported use of complementary and alternative medicine therapies in a reflexology randomized clinical trial. Alternative Therapies in Health and Medicine, 19(5), 31-37.

Zyoud, S.H., Al-Jabi, S.W., \& Sweileh, W.M. (2015). Scientific publications from Arab world in leading journals of Integrative and Complementary Medicine: A bibliometric analysis. BMC Complementary and Alternative Medicine, 15(1), 308.

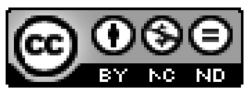

This is an open access article licensed under the Creative Commons Attribution-NonCommercialNoDerivs License (http://creativecommons.org/licenses/by-nc-nd/4.0/).

Journal of Data and Information Science

http://www.jdis.org https://www.degruyter.com/view/j/jdis 